Louisiana State University

LSU Digital Commons

Faculty Publications

Department of Physics \& Astronomy

$11-28-2018$

\title{
Tuning martensitic transitions in (MnNiSi)0.65(Fe2Ge)0.35 through heat treatment and hydrostatic pressure
}

Jing Han Chen

Louisiana State University

Ahmad Us Saleheen

Louisiana State University

Sunil K. Karna

Louisiana State University

David P. Young

Louisiana State University

Igor Dubenko

Southern Illinois University Carbondale

See next page for additional authors

Follow this and additional works at: https://digitalcommons.Isu.edu/physics_astronomy_pubs

\section{Recommended Citation}

Chen, J., Us Saleheen, A., Karna, S., Young, D., Dubenko, I., Ali, N., \& Stadler, S. (2018). Tuning martensitic transitions in (MnNiSi)0.65(Fe2Ge)0.35 through heat treatment and hydrostatic pressure. Journal of Applied Physics, 124 (20) https://doi.org/10.1063/1.5051551

This Article is brought to you for free and open access by the Department of Physics \& Astronomy at LSU Digital Commons. It has been accepted for inclusion in Faculty Publications by an authorized administrator of LSU Digital Commons. For more information, please contact ir@lsu.edu. 


\section{Authors}

Jing Han Chen, Ahmad Us Saleheen, Sunil K. Karna, David P. Young, Igor Dubenko, Naushad Ali, and Shane Stadler 


\section{Tuning martensitic transitions in $(\mathrm{MnNiSi})_{0.65}\left(\mathrm{Fe}_{2} \mathrm{Ge}\right)_{0.35}$ through heat treatment and hydrostatic pressure}

Cite as: J. Appl. Phys. 124, 203903 (2018); https://doi.org/10.1063/1.5051551

Submitted: 09 August 2018. Accepted: 01 November 2018 . Published Online: 28 November 2018

Jing-Han Chen, Ahmad Us Saleheen, Sunil K. Karna, David P. Young, Igor Dubenko, Naushad Ali, and Shane Stadler
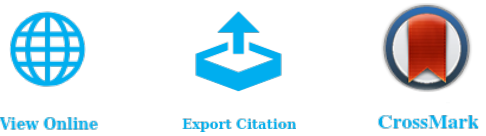

\section{ARTICLES YOU MAY BE INTERESTED IN}

Improved thermal stability in doped MnN/CoFe exchange bias systems

Journal of Applied Physics 124, 203902 (2018); https://doi.org/10.1063/1.5051584

Magnetostructural transition and magnetocaloric effect in $\mathrm{MnNiSi-Fe} 2 \mathrm{Ge}$ system

Applied Physics Letters 107, 212403 (2015); https://doi.org/10.1063/1.4936610

Barocaloric and magnetocaloric effects in (MnNiSi $)_{1-x}\left(\mathrm{FeCoGe}_{X}\right.$

Applied Physics Letters 112, 021907 (2018); https://doi.org/10.1063/1.5011743

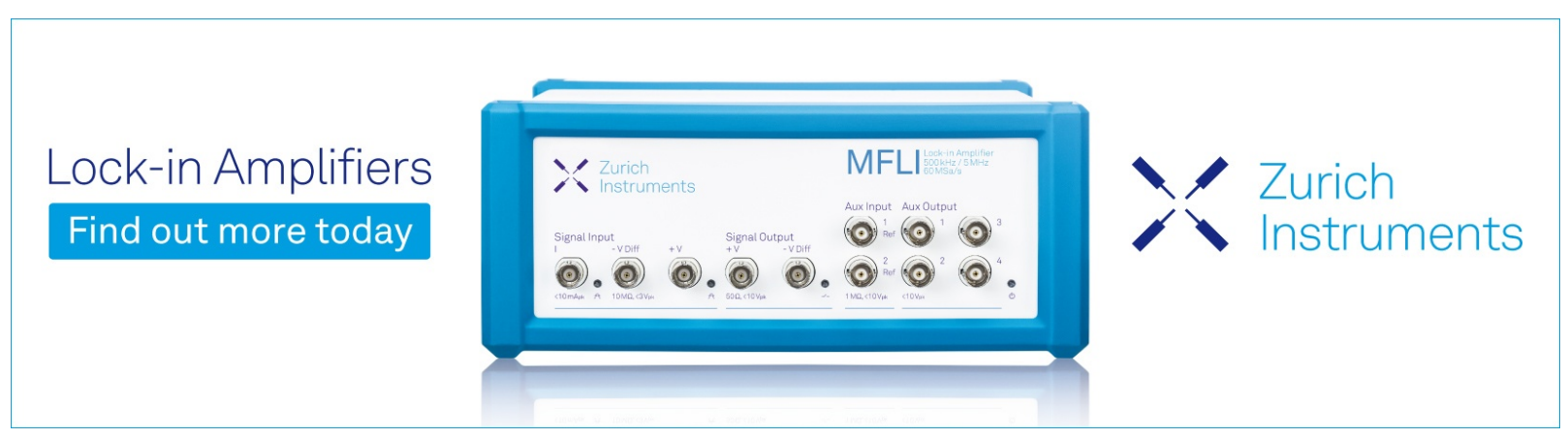




\title{
Tuning martensitic transitions in $(\mathrm{MnNiSi})_{0.65}\left(\mathrm{Fe}_{2} \mathrm{Ge}\right)_{0.35}$ through heat treatment and hydrostatic pressure
}

\author{
Jing-Han Chen, ${ }^{1, a)}$ Ahmad Us Saleheen, ${ }^{1}$ Sunil K. Karna, ${ }^{1}$ David P. Young, ${ }^{1}$ \\ Igor Dubenko, ${ }^{2}$ Naushad $\mathrm{Ali}^{2}{ }^{2}$ and Shane Stadler ${ }^{1}$ \\ ${ }^{1}$ Department of Physics and Astronomy, Louisiana State University, Baton Rouge, Louisiana 70803, USA \\ ${ }^{2}$ Department of Physics, Southern Illinois University, Carbondale, Illinois 62901, USA
}

(Received 9 August 2018; accepted 1 November 2018; published online 28 November 2018)

\begin{abstract}
A first-order magneto-structural transition from a ferromagnetic orthorhombic TiNiSi-type martensite phase to a paramagnetic hexagonal $\mathrm{Ni}_{2} \mathrm{In}$-type austenite phase was observed in $(\mathrm{MnNiSi})_{0.65}\left(\mathrm{Fe}_{2} \mathrm{Ge}\right)_{0.35}$. In this work, we demonstrate that the first-order magneto-structural transition temperature for a given composition is tunable over a wide temperature range through heat treatment and hydrostatic pressure. The first-order transition temperature was reduced by over $100 \mathrm{~K}$ as the annealing temperature went from 600 to $900^{\circ} \mathrm{C}$, and this first-order transition was converted to second order when the sample was annealed at $1000^{\circ} \mathrm{C}$. The maximum magnetic-induced isothermal entropy change with $\mu_{0} \Delta H=7 \mathrm{~T}$ reaches $-58 \mathrm{~J} / \mathrm{kg} \mathrm{K}$ for the sample annealed at $600{ }^{\circ} \mathrm{C}$, and the relative cooling power reaches $558 \mathrm{~J} / \mathrm{kg}$ for the sample annealed at $700{ }^{\circ} \mathrm{C}$. Similar to the influence of annealing temperatures, the first-order martensitic transition temperatures were reduced as the application of hydrostatic pressure increased until they were converted to second order. Our results suggest that the $(\mathrm{MnNiSi})_{0.65}\left(\mathrm{Fe}_{2} \mathrm{Ge}\right)_{0.35}$ system is a promising platform for tuning magneto-structural transitions and the associated magnetocaloric effects. Furthermore, a similar heat treatment methodology or application of hydrostatic pressure can be applied to MnNiSi-based shape memory alloys to tailor their working transition temperatures. Published by AIP Publishing. https://doi.org/10.1063/1.5051551
\end{abstract}

\section{INTRODUCTION}

Magnetic refrigeration based on the magnetocaloric effect (MCE) is generally considered as a potential substitution for conventional vapor compression systems due to its predicted high efficiency and environmental friendliness. ${ }^{1-8}$ The performance of MCE materials can be quantified as the magnetic entropy change in an isothermal process or the temperature change in an adiabatic process. ${ }^{9}$ The search for magnetocaloric materials for magnetic refrigeration applications has intensified since a giant $\mathrm{MCE}$ in $\mathrm{Gd}_{5}(\mathrm{Si}, \mathrm{Ge})_{4}$ with a first-order transition near room temperature was discovered. ${ }^{10}$

Intermetallic $\operatorname{Mn} T X(T=\mathrm{Co}, \mathrm{Ni}$ and $X=\mathrm{Ge}, \mathrm{Si})$ systems have drawn increasing attention recently due to their giant MCEs caused by first-order magneto-structural transitions from a $\mathrm{Ni}_{2}$ In-type hexagonal structure (space group: $P 6_{3} / m m c$ ) to a TiNiSi-type orthorhombic structure (space group: Pnma). ${ }^{11-13}$ Considerable interest has been drawn to the MnCoGe-based and MnNiGe-based systems in the context of MCEs because of their martensitic transitions near room temperature that can be tuned by stoichiometry modification, substitution of foreign atoms, doping with interstitial atoms, vacancy tuning, or pressure application. ${ }^{14-31}$ Regarding MnNiSi, single-element substitution is not sufficient to bring its extremely high structural transition temperature $(1200 \mathrm{~K})$ down to room temperature. ${ }^{32,33}$ Alternatively, chemically alloying MnNiSi with another ternary compound in a stable $\mathrm{Ni}_{2} \mathrm{In}$-type crystal structure, such as FeNiGe, CoNiGe, FeCoGe, or MnFeGe, has succeeded in reducing the first-order magneto-structural transitions to room

${ }^{\text {a)} E l e c t r o n i c ~ m a i l: ~ j h c h e n 10 @ 1 s u . e d u ~}$ temperature. ${ }^{34-37}$ Furthermore, these MnNiSi-based magnetocaloric materials are reported to be sensitive to minor variations in composition and hydrostatic pressure. ${ }^{36,34}$ Such properties provide additional degrees of freedom and thermodynamic flexibility for designing multifunctional applications.

It has been previously reported that the martensitic transition temperatures of $(\mathrm{MnNiSi})_{1-x}\left(\mathrm{Fe}_{2} \mathrm{Ge}\right)_{x}$ alloys shift from $490 \mathrm{~K}$ to $100 \mathrm{~K}$ as the degree of $\mathrm{Fe}_{2} \mathrm{Ge}$ substitution varies from $x=0.28$ to $x=0.38 .^{38,39}$ In the current work, polycrystalline samples of $(\mathrm{MnNiSi})_{0.65}\left(\mathrm{Fe}_{2} \mathrm{Ge}\right)_{0.35}$ were prepared and heat treated at temperatures ranging from $600{ }^{\circ} \mathrm{C}$ to $1000{ }^{\circ} \mathrm{C}$ before quenching. Their first-order martensitic transitions shifted to lower temperatures as the annealing/quenching temperatures increased from $600{ }^{\circ} \mathrm{C}$ to $900{ }^{\circ} \mathrm{C}$. The first-order martensitic transition was then converted to second order for an annealing/quenching temperature of $1000{ }^{\circ} \mathrm{C}$. The maximum magnetic entropy change $\left(\Delta S_{\max }\right)$ at $7 \mathrm{~T}$ was $-58 \mathrm{~J} / \mathrm{kg} \mathrm{K}$ for the sample annealed at $600^{\circ} \mathrm{C}$, and the relative cooling power estimated by the product of $\Delta S_{\max }$ and the full width at half maximum of the entropy $\delta_{F W H M}{ }^{40,41}$

$$
R C P=\Delta S_{\max } \times \delta_{F W H M}
$$

reaches $558 \mathrm{~J} / \mathrm{kg}$ for the sample annealed at $700{ }^{\circ} \mathrm{C}$.

\section{SAMPLE PREPARATION}

A 30-g (MnNiSi) $)_{0.65}\left(\mathrm{Fe}_{2} \mathrm{Ge}\right)_{0.35}$ (nominal composition) alloy ingot was prepared from high purity Ni $(99.995 \%), \mathrm{Mn}$ (99.95\%), Si (99.9999\%), Fe (99.99\%), and Ge (99.999\%) elemental components by melting in a RF induction furnace in an Ar atmosphere. After removing the sample from the 
TABLE I. The annealing temperature conditions of the samples and their corresponding properties. $\Delta S_{\max }$ was estimated from magnetization measurements. Relative cooling power, $R C P$, for $\mu_{0} \Delta H=7 \mathrm{~T}$ was estimated by using Eq. (1).

\begin{tabular}{|c|c|c|c|c|c|c|c|c|}
\hline Label & Annealing temperature $\left({ }^{\circ} \mathrm{C}\right)$ & $A_{s}(\mathrm{~K})$ & $A_{f}(\mathrm{~K})$ & $M_{s}(\mathrm{~K})$ & $M_{f}(\mathrm{~K})$ & $\Delta S_{\max }(\mathrm{J} / \mathrm{kg} \mathrm{K})$ & $R C P(\mathrm{~J} / \mathrm{kg})$ & $M_{\text {sat }} / f . u .\left(\mu_{B}\right)$ \\
\hline AQ1000 & 1000 (quench) & \multicolumn{6}{|c|}{$T_{C}=225.65 \mathrm{~K}$ (2nd order phase transition only) } & 2.238 \\
\hline AQ900 & 900 (quench) & 199.72 & 221.58 & 221.25 & 174.00 & -25 & 438 & 3.008 \\
\hline AQ800 & 800 (quench) & 190.95 & 237.99 & 221.28 & 177.79 & -24 & 471 & 3.057 \\
\hline AQ700 & 700 (quench) & 241.67 & 284.78 & 274.53 & 213.17 & -29 & 558 & 3.078 \\
\hline AQ600 & 600 (quench) & 294.45 & 301.87 & 284.11 & 278.22 & -58 & 442 & 3.362 \\
\hline SC1000 & 1000 (slow cool) & 327.62 & 331.83 & 316.86 & 308.32 & -45 & 483 & 3.234 \\
\hline
\end{tabular}

furnace, the ingot was cracked into irregularly shaped pieces. For subsequent (different) heat treatments, 3-5 g of the sample was melted again in the arc furnace to form a single and contiguous specimen and then sealed in a quartz tube under vacuum. All the samples in this report were fabricated from a single bulk master alloy. The nominal composition of the sample is $(\mathrm{MnNiSi})_{0.65}\left(\mathrm{Fe}_{2} \mathrm{Ge}\right)_{0.35}$. The final composition of the as-cast sample was measured by using the wavelength-dispersive spectrometry (WDS) equipped with the JEOL-8230 Superprobe and was found to be $\mathrm{Mn}_{0.72} \mathrm{Ni}_{0.59} \mathrm{Si}_{0.64} \mathrm{Fe}_{0.70} \mathrm{Ge}_{0.32}$. In order to confirm the compositional homogeneity of the as-cast sample, we performed the measurement in several different regions and the compositional variation between the grains was less than 0.08 at. $\%$, while the composition of annealed samples was found to be the same within this variation. The samples were annealed at $1000{ }^{\circ} \mathrm{C}, 900{ }^{\circ} \mathrm{C}, 800{ }^{\circ} \mathrm{C}, 700{ }^{\circ} \mathrm{C}$, and $600{ }^{\circ} \mathrm{C}$ for 5 days (labeled as AQ1000, AQ900, AQ800, AQ700, and AQ600, respectively) and then immediately quenched in ice water. One piece of the as-cast ingot (labeled as $\mathrm{SC} 1000$ ) was slowly cooled from $1000^{\circ} \mathrm{C}$ for comparison with the quenched samples. The preparation parameters and corresponding properties of the samples used in this work are summarized in Table I.

\section{THERMAL ANALYSIS}

A simultaneous differential scanning calorimetry (DSC) and thermogravimetric analysis (TGA) (SDT Q600) manufactured by TA Instruments, Inc. was employed to measure the heat flow as a function of temperature for the as-cast ingot. The temperature was scanned from $50{ }^{\circ} \mathrm{C}$ to $1400{ }^{\circ} \mathrm{C}$ back and forth twice in order to confirm reproducibility, as shown in Fig. 1. The first-order phase transition with thermal hysteresis observed from $1050{ }^{\circ} \mathrm{C}$ to $1130{ }^{\circ} \mathrm{C}$ (in Fig. 1) corresponds to the melting point of the as-cast ingot, and no other transitions were observed before the ingot melted. Therefore, the ingot presumably stabilizes in the same crystal structure above $50{ }^{\circ} \mathrm{C}$ all the way up to the melting temperature. Taking this thermal analysis as a guideline, we then annealed the samples at selected temperatures between 1000 ${ }^{\circ} \mathrm{C}$ and $600{ }^{\circ} \mathrm{C}$ before quenching or slow cooling. Since the samples presumably all stabilize in the same crystal structure under these annealing conditions, it was expected that the property variations among them (shown later) come from variations in atomic ordering within the same crystal structure.

\section{X-RAY DIFFRACTION}

To further investigate the crystal structures of these samples, powder X-ray diffraction (XRD) was performed using a Scintag XDS2000 powder diffractometer with $\mathrm{Cu} \mathrm{K} \alpha$ radiation at room temperature. Since these materials are sensitive to pressure, ${ }^{36,34}$ instead of grinding the samples, the powder samples used in XRD measurements were prepared by temperature cycling the samples through their transitions until they spontaneously broke into powders. Figure 2 shows the $\mathrm{XRD}$ results for all the samples at room temperature.

Figure 2 shows that sample AQ1000 stabilizes in the $\mathrm{Ni}_{2}$ In-type hexagonal structure, and the fraction of the TiNiSi-type orthorhombic phase increases with decreasing annealing temperature. It is known that the TiNiSi-type structure can be regarded as an orthorhombic distortion of the $\mathrm{Ni}_{2}$ In-type hexagonal structure. The lattice parameters are related as $a_{\text {orth }}=c_{\text {hex }}, b_{\text {orth }}=a_{\text {hex }}$, and $c_{\text {orth }}=\sqrt{3} a_{\text {hex }} .{ }^{38}$ The lattice constants and volumes from the Rietveld refinements using General Structure Analysis System (GSAS) software s2-44 $^{4}$ are tabulated in Table II. The volumes per formula unit and lattice constants at room temperature increase as the annealing temperature decreases.

Although the XRD patterns for all the samples point to the same crystal structures for the martensite and austenite phases, we have observed a variation of the martensitic transition temperatures by over $100 \mathrm{~K}$ from the magnetization data presented later. Hence, the variation of the martensitic transitions among samples presumably comes from the variation of atomic

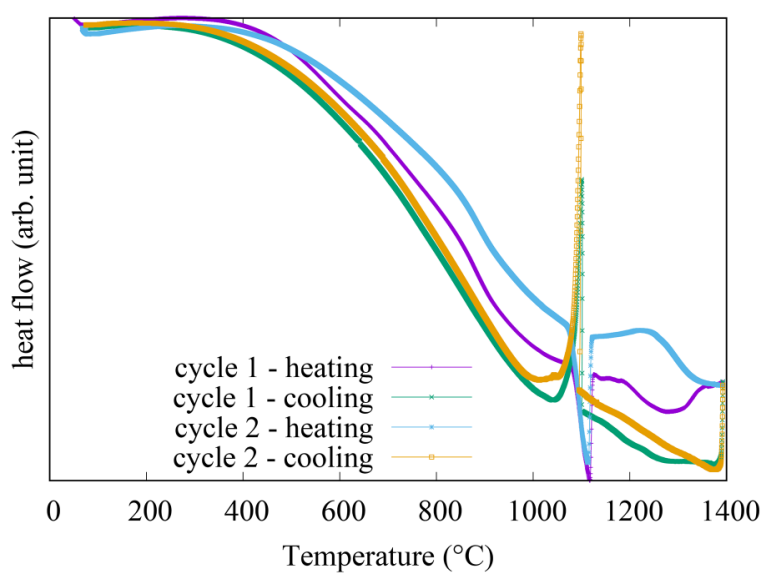

FIG. 1. The heat flow data of the as-cast ingot obtained from DSC measurements. The ingot melted around $1100{ }^{\circ} \mathrm{C}$, and no first-order structural transitions were observed before it melted. 


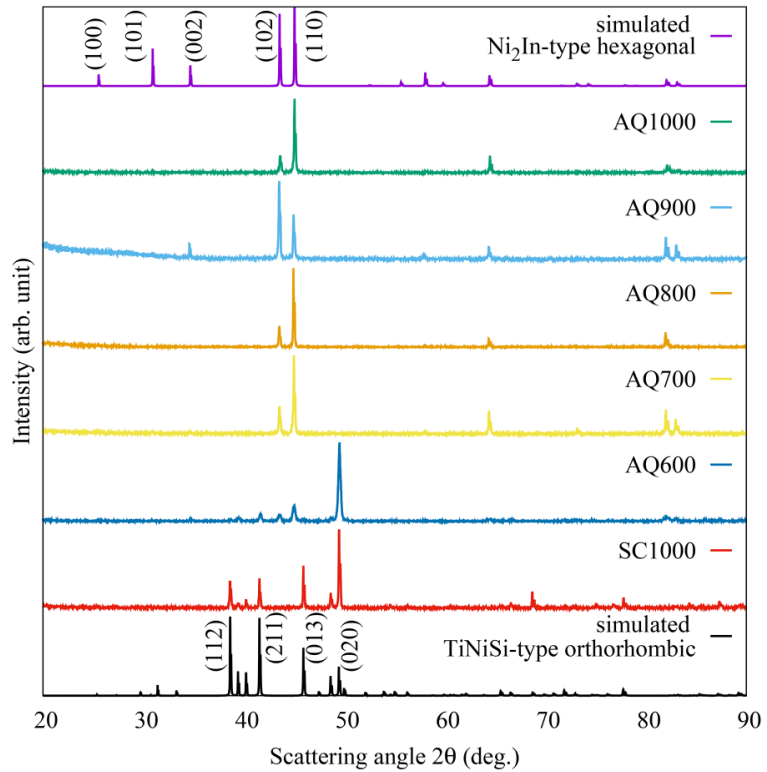

FIG. 2. $\mathrm{Cu} \mathrm{K} \alpha$ powder X-ray diffraction patterns of all samples at room temperature. The bottom panel shows the simulated TiNiSi-type orthorhombic pattern, and the top panel shows the simulated $\mathrm{Ni}_{2}$ In-type hexagonal pattern.

surroundings (which is often not easily detectable in XRD measurements) induced by different thermal heat treatments.

To further investigate the thermo-induced structural transition in our samples, temperature-dependent XRD measurements of samples AQ900 and SC1000 were performed at the X-ray Science Division beamlines at the Advanced Photon Source, Argonne National Laboratory using an X-ray wavelength of $0.41 \AA$. The XRD patterns for both samples SC1000 and AQ900 show the $\mathrm{Ni}_{2}$ In-type hexagonal martensite phase at low temperature and the TiNiSi-type orthorhombic austenite phase at high temperatures. Representative results for sample AQ900 in the temperature region of the martensite $(160 \mathrm{~K})$ and austenite phases $(240 \mathrm{~K})$ are shown in Fig. 3. The XRD patterns were analyzed using the GSAS program following the Rietveld profile refining method. ${ }^{42-44}$ The XRD refinements at $240 \mathrm{~K}$ and $160 \mathrm{~K}$ were carried out assuming a hexagonal structure (space group: $P 6_{3} / m m c$ ) and an orthorhombic structure (space group: Pnma), respectively. The structural parameters obtained from the refinements are summarized in Table III. Figure 3 displays the observed (circles) and fitted (solid red lines) diffraction patterns. Their differences are plotted at the bottom along with the calculated Bragg reflections positions (solid vertical lines).

TABLE II. The lattice constants and cell volumes from the XRD refinement of room temperature powder diffraction data.

\begin{tabular}{lccccc}
\hline \hline Label & Major phase & $a(\AA)$ & $b(\AA)$ & $c(\AA)$ & vol./f.u. $\left(\AA^{3}\right)$ \\
\hline AQ1000 & $\mathrm{Ni}_{2}$ In & 4.023 & 4.023 & 5.161 & 36.164 \\
AQ900 & $\mathrm{Ni}_{2}$ In & 4.030 & 4.030 & 5.173 & 36.375 \\
AQ800 & $\mathrm{Ni}_{2}$ In & 4.031 & 4.031 & 5.176 & 36.422 \\
AQ700 & $\mathrm{Ni}_{2}$ In & 4.034 & 4.034 & 5.180 & 36.519 \\
AQ600 & $\mathrm{TiNiSi}$ & 5.821 & 3.685 & 6.879 & 36.896 \\
SC1000 & TiNiSi & 5.825 & 3.685 & 7.015 & 37.642 \\
\hline
\end{tabular}

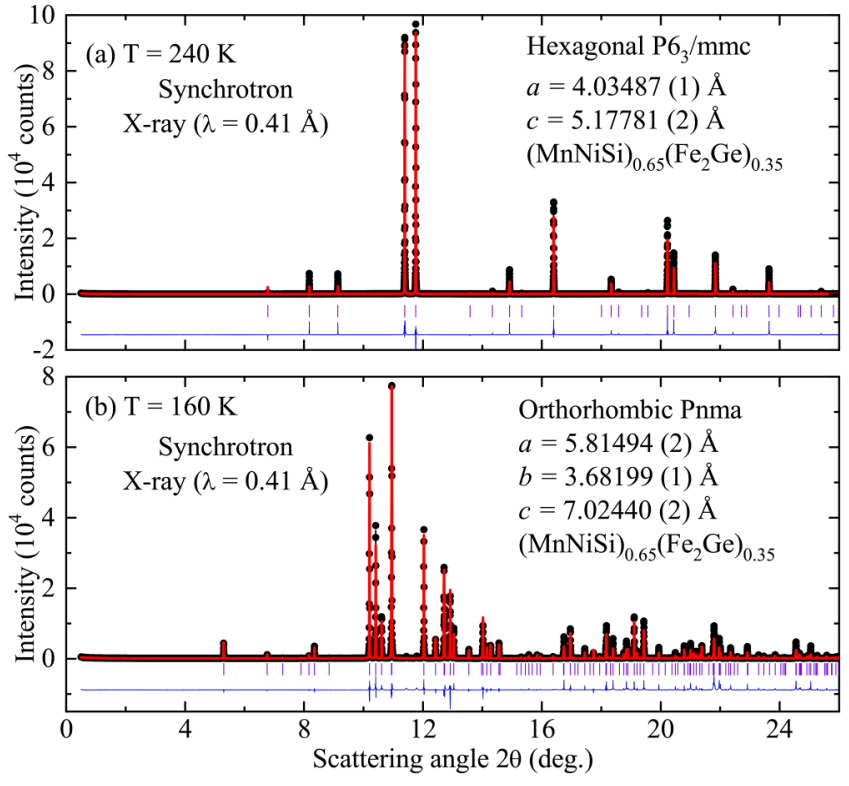

FIG. 3. X-ray diffraction patterns with (a) $T=240 \mathrm{~K}$ and (b) $T=160 \mathrm{~K}$ for sample AQ900 are shown with the observed (circles) and fitted (solid lines) diffraction patterns. Their differences plotted at the bottom along with the calculated Bragg reflection positions (solid vertical lines).

The temperature dependences of the unit cell parameters obtained through temperature-dependent XRD measurements are shown in Fig. 4. As the temperature increases, the TiNiSi-type orthorhombic martensite phase at low temperature was distorted with a reduced $a_{\text {orth }}$ and expanded $b_{\text {orth }}$ and was consequently stabilized in the $\mathrm{Ni}_{2} \mathrm{In}$-type hexagonal austenite phase at high temperatures. It is worth noting that the changes in cell parameters across the martensitic transition for samples AQ900 and SC1000 are quantitatively the same, while their transition temperatures are separated by over $100 \mathrm{~K}$.

\section{MAGNETIZATION MEASUREMENTS AND ANALYSIS}

The magnetization measurements were performed using a Magnetic Property Measurement System (MPMS)

TABLE III. Refined structural parameters for sample AQ900 at $160 \mathrm{~K}$ and $240 \mathrm{~K}$.

$$
T=240 \mathrm{~K}
$$

Hexagonal $P 6_{3} / m m c$ (space group No. 194) $a=b=4.03487(1) \AA, c=5.17781(2) \AA$

\begin{tabular}{lccccc}
\hline Atom & $\mathrm{x}$ & $\mathrm{y}$ & $\mathrm{z}$ & $\mathrm{M}$ & Occupancy \\
\hline $\mathrm{Mn} / \mathrm{Fe} 1$ & 0 & 0 & 0 & $2 \mathrm{a}$ & $0.65 / 0.35$ \\
$\mathrm{Ni} / \mathrm{Fe} 2$ & $2 / 3$ & $1 / 3$ & $1 / 4$ & $2 \mathrm{c}$ & $0.65 / 0.35$ \\
$\mathrm{Si} / \mathrm{Ge}$ & $1 / 3$ & $2 / 3$ & $1 / 4$ & $2 \mathrm{~d}$ & $0.65 / 0.35$ \\
& \multicolumn{7}{c}{$T=160 \mathrm{~K}$}
\end{tabular}

Orthorhombic Pnma (space group No. 62) $a=5.81494(2) \AA, b=3.68199(2) \AA, c=7.02440(1) \AA$

\begin{tabular}{lccccc}
\hline Atom & $\mathrm{x}$ & $\mathrm{y}$ & $\mathrm{z}$ & $\mathrm{M}$ & Occupancy \\
\hline $\mathrm{Mn} / \mathrm{Fe} 1$ & 0.0347 & 14 & 0.1813 & $4 \mathrm{c}$ & $0.65 / 0.35$ \\
$\mathrm{Ni} / \mathrm{Fe} 2$ & 0.1517 & 14 & 0.5566 & $4 \mathrm{c}$ & $0.65 / 0.35$ \\
$\mathrm{Si} / \mathrm{Ge}$ & 0.7538 & 14 & 0.6255 & $4 \mathrm{c}$ & $0.65 / 0.35$ \\
\hline
\end{tabular}



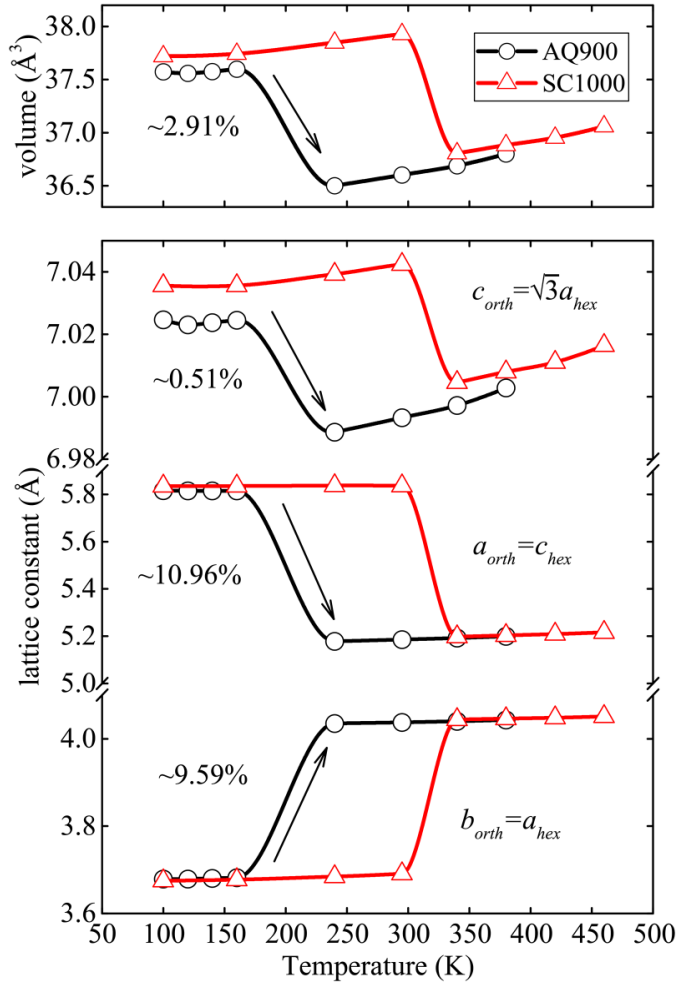

FIG. 4. Lattice constants and cell volumes versus temperature for samples AQ900 and SC1000. Note that changes in cell parameters across the martensitic transition for samples AQ900 and SC1000 are quantitatively the same, while their transition temperatures are separated by over $100 \mathrm{~K}$.

manufactured by Quantum Design within the temperature interval $10-400 \mathrm{~K}$ and in applied magnetic fields up to $7 \mathrm{~T}$. The isofield, temperature-dependent magnetization measurements were performed using zero-field cooled warming,

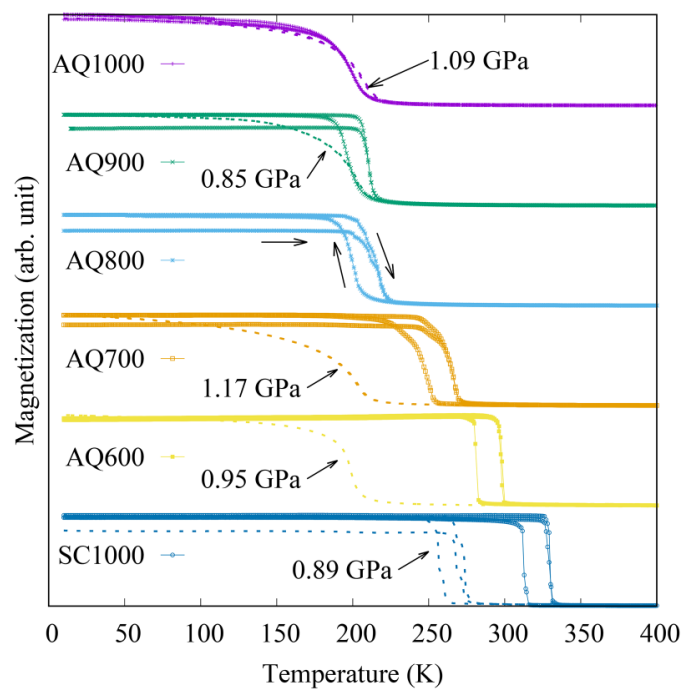

FIG. 5. The temperature-dependent magnetization of all samples in an applied field of $\mu_{0} H=0.1 \mathrm{~T}$ (solid lines: without pressures; dashed lines: with pressures) measured using zero-field cooled warming, field-cooled cooling, and field-cooled warming protocols. The arrows shown for sample AQ800 represent the direction of measurements. With increasing annealing temperature or hydrostatic pressure, the martensitic transition temperatures shifted to lower temperature.

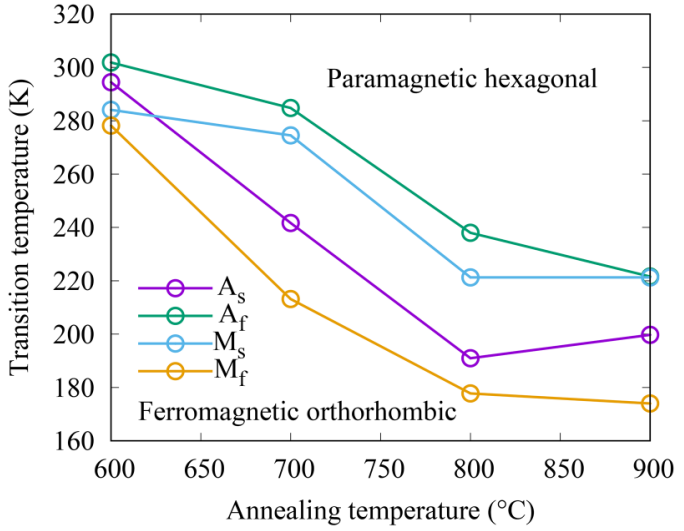

FIG. 6. The martensitic transition temperatures from magnetization measurements $M(T)$ vs. annealing temperature.

field-cooled cooling, and field-cooled warming protocols, and the results are shown in Fig. 5.

First-order martensitic transitions with thermal hysteresis were observed, and the transition temperatures decrease as the annealing temperature increases up to $900{ }^{\circ} \mathrm{C}$. For sample AQ1000, which was quenched at $1000{ }^{\circ} \mathrm{C}$, the first-order martensitic transition was completely suppressed, and only a second-order magnetic transition was observed. The start and finish temperatures of the martensitic transition $\left(A_{s}\right.$ : austenite start, $A_{f}$ : austenite finish, $M_{s}$ : martensite start, $M_{f}$ : martensite finish) were estimated from the iso-field magnetization measurements at $0.1 \mathrm{~T}$ and are listed in Table I. Figure 6 shows the near-linear dependence of the martensitic transition temperatures with the annealing temperatures, which is similar to that reported for $\mathrm{MnCoGe}$-based materials. ${ }^{45}$ Isothermal magnetization measurements at $T=4.2 \mathrm{~K}$ were performed in order to investigate the magnetization saturation and are shown in Fig. 7.

The saturation magnetization $\left(M_{s a t}\right)$ values in Table I were estimated by fitting the experimental magnetization data in high magnetic field using the law of approach-to-saturation ${ }^{46,47}$

$$
M=M_{s a t}\left(1-\frac{a}{H^{2}}-\frac{b}{H^{3}}\right),
$$

where $a$ and $b$ are fitting parameters. The sample AQ1000

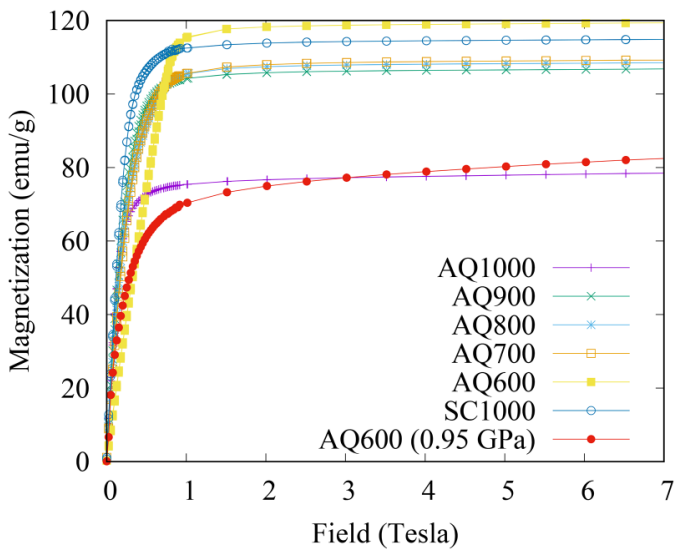

FIG. 7. The isothermal magnetization data of all samples at $T=4.2 \mathrm{~K}$. 
TABLE IV. The magnetic moments of $\mathrm{MnNiSi}$ and $\mathrm{Fe}_{2} \mathrm{Ge}$ per formula unit cell were obtained through DFT calculations using the WIEN2k software package.

\begin{tabular}{lcc}
\hline \hline Composition & Space group & $M_{\text {sat }}\left(\mu_{B}\right)$ \\
\hline MnNiSi & $P 6_{3} / m m c$ (hexagonal) & 2.647 \\
& $P n m a$ (orthorhombic) & 2.884 \\
$\mathrm{Fe}_{2} \mathrm{Ge}$ & $P 6_{3} / m m c$ (hexagonal) & 3.577 \\
& Pnma (orthorhombic) & 3.923 \\
\hline \hline
\end{tabular}

does not undergo a first-order martensitic transition and therefore stabilizes in the $\mathrm{Ni}_{2}$ In-type hexagonal crystal structure at $4.2 \mathrm{~K}$, where the others stabilize in the TiNiSi-type orthorhombic structure. Therefore, the abrupt decrease of $M_{\text {sat }}$ in sample AQ1000 is due to the difference between magnetic orthorhombic and hexagonal structures.

Since there are no experimental magnetization results available for single crystals of $\mathrm{Fe}_{2} \mathrm{Ge}$ as far as the authors are aware, density functional theory (DFT) calculations were performed using the WIEN2k software package, which is based on the full-potential augmented plane-wave plus local orbitals method. ${ }^{48}$ The goal was to understand the origin of the difference between magnetic saturation moments of the TiNiSi-type orthorhombic and the $\mathrm{Ni}_{2}$ In-type hexagonal structures. The saturation magnetic moments of $\mathrm{MnNiSi}$ and $\mathrm{Fe}_{2} \mathrm{Ge}$ for both the hexagonal and orthorhombic structures were calculated based on atomic positions taken from Table III. The moments of the hexagonal crystal structure in both $\mathrm{MnNiSi}$ and $\mathrm{Fe}_{2} \mathrm{Ge}$ appear to be lower than those of the orthorhombic crystal structure as summarized in Table IV. Therefore, it is reasonable that the saturation magnetization of AQ1000 is lower than the others since this sample only forms in the hexagonal structure. It is also interesting to note that the experimental saturation magnetization of our alloyed compounds falls close to those of $\mathrm{MnNiSi}$ and $\mathrm{Fe}_{2} \mathrm{Ge}$ from DFT calculations.

Magnetic measurements under hydrostatic pressure were performed using a commercial $\mathrm{BeCu}$ cylindrical pressure cell manufactured by Quantum Design. Daphne 7373 oil was used as the pressure transmitting medium. The magnitude of the applied pressure was calibrated by measuring the shift of the superconducting transition temperature of $\mathrm{Pb}$, which was placed in the cell with the sample as a reference manometer. Figure 5 (dashed lines) shows the effects of pressure on the martensitic transitions for samples AQ1000 (1.09 GPa), AQ900 (0.85 GPa), AQ700 (1.17 GPa), AQ600 $(0.95 \mathrm{GPa})$, and $\mathrm{SC} 1000(0.89 \mathrm{GPa})$ under the specified hydrostatic pressures. With the specified hydrostatic pressures, the first-order martensitic transition of samples AQ900, AQ700, and AQ600 was completely suppressed and only secondorder magnetic transitions were observed. For sample SC1000 under $0.89 \mathrm{GPa}$, the first-order martensitic transition remained, but the transition temperature was reduced. It is reasonable to expect the disappearance of the first-order martensitic transition in SC1000 if the applied pressure is large enough. However, this applied pressure $(0.89 \mathrm{GPa})$ in SC1000 is close to the upper limit of hydrostatic pressures allowable by the cell. Overall, the martensitic transition temperatures were reduced with the increase of hydrostatic pressures until they were completely converted to secondorder magnetic transitions, which is similar to the effects of increasing annealing temperatures. It is worth noting that, without martensitic transitions, the crystal remains in a $\mathrm{Ni}_{2}$ In-type hexagonal structure throughout our measurement temperatures. Therefore, the saturation magnetic moment of sample AQ600 under $0.95 \mathrm{GPa}$ at $4.2 \mathrm{~K}$ shown in Fig. 7 is reasonably close to the magnitude of sample AQ1000 at ambient pressure since the samples possess only $\mathrm{Ni}_{2}$ In-type hexagonal structures in both cases.

\section{MAGNETIC-INDUCED ISOTHERMAL ENTROPY}

The magnetic entropy change was estimated from isothermal magnetization data using the thermodynamic relation

$$
\begin{aligned}
& \Delta S(T, 0 \rightarrow H)=\frac{\partial}{\partial T}\left(\int_{0}^{H} M\left(T, H^{\prime}\right) d H^{\prime}\right)_{T=\text { const. }} \\
& \quad \cong \frac{1}{\Delta T}\left[\int_{0}^{H} M\left(T+\Delta T, H^{\prime}\right) d H^{\prime}-\int_{0}^{H} M\left(T, H^{\prime}\right) d H^{\prime}\right] .
\end{aligned}
$$

Therefore, a series of isothermal magnetization experiments were performed to obtain $M(T=$ const., $H)$. In order to eliminate a potential residual ferromagnetic martensite phase generated in each isothermal magnetization measurement, which may result in inaccurate entropy changes, the samples were heated to the complete paramagnetic austenite phase and then cooled down to the target measurement temperature under zero field before starting each isothermal magnetization measurement. The magnetic entropy change determined using this thermodynamic reset protocol should represent the magnetic entropy change in the direction from the austenite phase to the martensite phase. This protocol was referred to as the cooling protocol or the loop process in Refs. 49 and 50, because the results obtained using this protocol can also be revealed by calorimetric experiments during the cooling process. The change in magnetic entropy for a 7-T field is shown in Fig. 8 for each sample.

The quantitative values of the maximum magnetic entropy change and relative cooling power are listed in Table I.

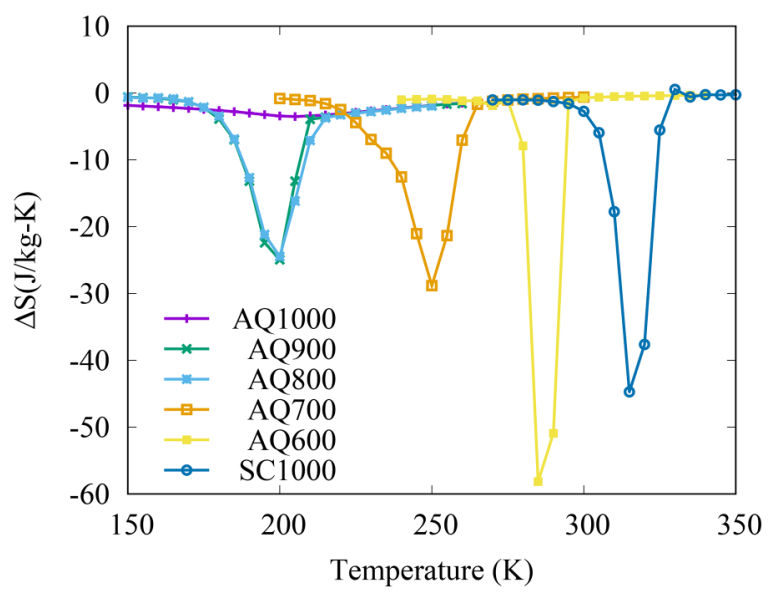

FIG. 8. The magnetic entropy changes for a 7-T field change of all samples estimated from magnetization isotherm measurements. 
Sample AQ1000 shows the smallest entropy change $\left(\Delta S_{\max }=-3.52 \mathrm{~J} / \mathrm{kg} \mathrm{K}\right.$ in 7-T field), since it only undergoes a second-order phase transition, while sample AQ600 shows the maximum magnetic entropy change $\left(\Delta S_{\max }=-58 \mathrm{~J} / \mathrm{kg} \mathrm{K}\right.$ in 7-T field) and sample AQ700 shows the maximum relative cooling power $(R C P=558 \mathrm{~J} / \mathrm{kg}$ for a field change of $7 \mathrm{~T})$.

\section{CONCLUSIONS}

In conclusion, the structural and magnetic properties of $(\mathrm{MnNiSi})_{0.65}\left(\mathrm{Fe}_{2} \mathrm{Ge}\right)_{0.35}$ under different annealing conditions and hydrostatic pressures were investigated. First-order martensitic transitions from a $\mathrm{Ni}_{2} \mathrm{In}$-type hexagonal austenite phase (space group: $\mathrm{PG}_{3} / \mathrm{mmc}$ ) to a TiNiSi-type orthorhombic martensite phase (space group: Pnma) were observed, and the martensitic transition temperatures varied in a wide temperature range by modifying either the annealing temperatures or hydrostatic pressures. As the annealing temperature increased from $600{ }^{\circ} \mathrm{C}$ to $900{ }^{\circ} \mathrm{C}$, the martensitic transition temperatures decreased. However, for an annealing temperature equal to $1000^{\circ} \mathrm{C}$, the first-order martensitic transition was completely suppressed, and only a secondorder magnetic transition was observed. Similar to the effect of increasing annealing temperatures, the first-order martensitic transitions were suppressed as the applied hydrostatic pressure increased until they were completely converted to a $\mathrm{Ni}_{2} \mathrm{In}$-type hexagonal structure with only second-order magnetic transitions. Such a variation of the martensitic transition temperature suggests a clear route for tuning the working temperature of the materials for magnetic refrigeration applications by modifying the heat treatment or by applying hydrostatic pressures.

\section{ACKNOWLEDGMENTS}

Shane Stadler acknowledges support from the U.S. Department of Energy (DOE), Office of Basic Energy Sciences under Award No. DE-FG02-13ER46946. Naushad Ali and Igor Dubenko acknowledge support from the U.S. Department of Energy (DOE), Office of Basic Energy Sciences under Award No. DE-FG02-06ER46291. David P. Young acknowledges support from the U.S. National Science Foundation (NSF), Division of Materials Research under Award No. NSF-DMR1306392. Portions of this research were conducted with high performance computing resources provided by Louisiana State University (http://www.hpc.lsu.edu). The DSC data used in this publication were collected at the Center for Advanced Microstructures and Devices (CAMD) under the supervision of Dr. Amitava Roy. This research used resources of the Advanced Photon Source, a U.S. Department of Energy (DOE) Office of Science User Facility operated for the DOE Office of Science by the Argonne National Laboratory under Contract No. DE-AC02-06CH11357. The XRD data analysis was carried out by S. K. Karna who acknowledged support of the U.S. Department of Energy (DOE), Office of Science, Basic Energy Sciences, under EPSCoR Grant No. DE-SC0012432 with additional support from the Louisiana Board of Regents.
${ }^{1}$ E. Brück, O. Tegus, D. C. Thanh, N. T. Trung, and K. H. J. Buschow, Int. J. Refrig. 31, 763 (2008).

${ }^{2}$ K. G. Sandeman, Scr. Mater. 67, 566 (2012).

${ }^{3}$ E. Brück, J. Phys. D 38, R381 (2005).

${ }^{4}$ B. G. Shen, J. R. Sun, F. X. Hu, H. W. Zhang, and Z. H. Cheng, Adv. Mater. 21, 4545 (2009).

${ }^{5}$ K. A. Gschneidner, Jr. and V. K. Pecharsky, Int. J. Refrig. 31, 945 (2008).

${ }^{6}$ K. A. Gschneidner, Jr., V. K. Pecharsky, and A. O. Tsokol, Rep. Prog. Phys. 68, 1479 (2005).

${ }^{7}$ V. Franco, J. S. Blázquez, B. Ingale, and A. Conde, Annu. Rev. Mater. Res. 42, 305 (2012).

${ }^{8}$ T. Gottschall, A. Gràcia-Condal, M. Fries, A. Taubel, L. Pfeuffer, L. Mañosa, A. Planes, K. P. Skokov, and O. Gutfleisch, Nat. Mater. 17, 929 (2018).

${ }^{9}$ V. K. Pecharsky and K. A. Gschneidner, Jr., J. Appl. Phys. 86, 565 (1999).

${ }^{10}$ V. K. Pecharsky and K. A. Gschneidner, Jr., Phys. Rev. Lett. 78, 4494 (1997).

${ }^{11}$ W. Bażela, A. Szytuła, J. Todorović, Z. Tomkowicz, and A. Zięba, Phys. Status Solidi A 38, 721 (1976).

${ }^{12}$ H. Fjellvåg and A. F. Andresen, J. Magn. Magn. Mater. 50, 291 (1985).

${ }^{13}$ T. Kanomata, H. Ishigaki, T. Suzuki, H. Yoshida, S. Abe, and T. Kaneko, J. Magn. Magn. Mater. 140-144, 131 (1995).

${ }^{14}$ T. Samanta, P. Lloveras, A. Us Saleheen, D. L. Lepkowski, E. Kramer, I. Dubenko, P. W. Adams, D. P. Young, M. Barrio, J. L. Tamarit, N. Ali, and S. Stadler, Appl. Phys. Lett. 112, 021907 (2018).

${ }^{15}$ C. L. Zhang, D. H. Wang, Q. Q. Cao, Z. D. Han, H. C. Xuan, and Y. W. Du, Appl. Phys. Lett. 93, 122505 (2008).

${ }^{16}$ E. Liu, Y. Du, J. Chen, W. Wang, H. Zhang, and G. Wu, IEEE Trans. Magn. 47, 4041 (2011).

${ }^{17}$ E. Liu, W. Wang, L. Feng, W. Zhu, G. Li, J. Chen, H. Zhang, G. Wu, C. Jiang, H. Xu, and F. de Boer, Nat. Commun. 3, 873 (2012).

${ }^{18}$ E. K. Liu, H. G. Zhang, G. Z. Xu, X. M. Zhang, R. S. Ma, W. H. Wang, J. L. Chen, H. W. Zhang, G. H. Wu, L. Feng, and X. X. Zhang, Appl. Phys. Lett. 102, 122405 (2013).

${ }^{19}$ T. Samanta, I. Dubenko, A. Quetz, S. Temple, S. Stadler, and N. Ali, Appl. Phys. Lett. 100, 052404 (2012).

${ }^{20}$ J. B. A. Hamer, R. Daou, S. Özcan, N. D. Mathur, D. J. Fray, and K. G. Sandeman, J. Magn. Magn. Mater. 321, 3535 (2009).

${ }^{21}$ N. T. Trung, V. Biharie, L. Zhang, L. Caron, K. H. J. Buschow, and E. Brück, Appl. Phys. Lett. 96, 162507 (2010).

${ }^{22}$ N. T. Trung, L. Zhang, L. Caron, K. H. J. Buschow, and E. Brück, Appl. Phys. Lett. 96, 172504 (2010).

${ }^{23}$ T. Samanta, I. Dubenko, A. Quetz, S. Stadler, and N. Ali, Appl. Phys. Lett. 101, 242405 (2012).

${ }^{24}$ J. Liu, K. Skokov, and O. Gutfleisch, Scr. Mater. 66, 642 (2012).

${ }^{25}$ D. Choudhury, T. Suzuki, Y. Tokura, and Y. Taguchi, Sci. Rep. 4, 7544 (2014).

${ }^{26}$ E. K. Liu, W. Zhu, L. Feng, J. L. Chen, W. H. Wang, G. H. Wu, H. Y. Liu, F. B. Meng, H. Z. Luo, and Y. X. Li, Europhys. Lett. 91, 17003 (2010).

${ }^{27}$ C. L. Zhang, H. F. Shi, E. J. Ye, Y. G. Nie, Z. D. Han, and D. H. Wang, J. Alloys Compd. 639, 36 (2015).

${ }^{28}$ D. Zhang, Z. Nie, Z. Wang, L. Huang, Q. Zhang, and Y. dong Wang, J. Magn. Magn. Mater. 387, 107 (2015).

${ }^{29}$ S. C. Ma, Y. X. Zheng, H. C. Xuan, L. J. Shen, Q. Q. Cao, D. H. Wang, Z. C. Zhong, and Y. W. Du, J. Magn. Magn. Mater. 324, 135 (2012).

${ }^{30}$ Y. K. Fang, C. C. Yeh, C. W. Chang, W. C. Chang, M. G. Zhu, and W. Li, Scr. Mater. 57, 453 (2007).

${ }^{31}$ J.-T. Wang, D.-S. Wang, C. Chen, O. Nashima, T. Kanomata, H. Mizuseki, and Y. Kawazoe, Appl. Phys. Lett. 89, 262504 (2006).

${ }^{32}$ W. Bażela, A. Szytuła, J. Todorović, and A. Zięba, Phys. Status Solidi A 64, 367 (1981).

${ }^{33}$ V. Johnson, Inorg. Chem. 14, 1117 (1975).

${ }^{34}$ T. Samanta, D. L. Lepkowski, A. U. Saleheen, A. Shankar, J. Prestigiacomo, I. Dubenko, A. Quetz, I. W. H. Oswald, G. T. McCandless, J. Y. Chan, P. W. Adams, D. P. Young, N. Ali, and S. Stadler, Phys. Rev. B 91, 020401 (2015).

${ }^{35}$ J. Liu, Y. Gong, G. Xu, G. Peng, I. A. Shah, N. ul Hassan, and F. Xu, Sci. Rep. 6, 23386 (2016).

${ }^{36}$ T. Samanta, D. L. Lepkowski, A. U. Saleheen, A. Shankar, J. Prestigiacomo, I. Dubenko, A. Quetz, I. W. H. Oswald, G. T. McCandless, J. Y. Chan, P. W. Adams, D. P. Young, N. Ali, and S. Stadler, J. Appl. Phys. 117, 123911 (2015).

${ }^{37}$ C. L. Zhang, D. H. Wang, Z. D. Han, B. Qian, H. F. Shi, C. Zhu, J. Chen, and T. Z. Wang, Appl. Phys. Lett. 103, 132411 (2013). 
${ }^{38}$ C. L. Zhang, H. F. Shi, E. J. Ye, Y. G. Nie, Z. D. Han, B. Qian, and D. H. Wang, Appl. Phys. Lett. 107, 212403 (2015).

${ }^{39}$ K. Deepak and R. Ramanujan, J. Alloys Compd. 743, 494 (2018).

${ }^{40}$ K. A. Gschneidner and V. K. Pecharsky, Annu. Rev. Mater. Sci. 30, 387 (2000).

${ }^{41}$ L. D. Griffith, Y. Mudryk, J. Slaughter, and V. K. Pecharsky, J. Appl. Phys. 123, 034902 (2018).

${ }^{42}$ A. C. Larson and R. B. V. Dreele, Los Alamos National Laboratory Report LAUR 86, 1994.

${ }^{43}$ B. H. Toby, J. Appl. Cryst. 34, 210 (2001).

${ }^{44}$ H. M. Rietveld, J. Appl. Cryst. 2, 65 (1969).
${ }^{45}$ A. Quintana-Nedelcos, J. L. Sánchez Llamazares, and H. Flores-Zu niga, J. Alloys Compd. 644, 1003 (2015).

${ }^{46}$ A. H. Morrish, The Physical Principles of Magnetism (Wiley, 2001), Chap. 7.

${ }^{47}$ B. D. Cullity and C. D. Graham, Introduction to Magnetic Materials (Wiley, 2011), Chap. 9

${ }^{48}$ K. Schwarz, P. Blaha, and G. Madsen, Comput. Phys. Commun. 147, 71 (2002).

${ }^{49}$ J.-H. Chen, A. Us Saleheen, P. W. Adams, D. P. Young, N. Ali, and S. Stadler, J. Appl. Phys. 123, 145101 (2018).

${ }^{50}$ L. Caron, Z. Q. Ou, T. T. Nguyen, D. T. C. Thanh, O. Tegus, and E. Brück, J. Magn. Magn. Mater. 321, 3559 (2009). 